\title{
Stress impedes exploration and the acquisition of spatial information in the eight-arm radial maze
}

\author{
TRACEY J. SHORS and ERIC DRYVER \\ Princeton University, Princeton, New Jersey
}

\begin{abstract}
To determine whether stress impairs the acquisition of spatial information in the eight-arm radial maze, one group of rats $(n=8)$ was restrained and exposed to ninety 1-sec, 1-mA tailshocks. Twenty-four hours later, they were reexposed to 5 shocks, and 30 min later, they were tested on the radial maze with the use of obvious spatial cues. Compared with the unstressed controls $(n=8)$, the stressed rats displayed a significantly different distribution of errors across the 12 trials, an effect attributable, in part, to their initial decrease in exploration and increase in perseveration. An equation that controls for the changing probability of choices and errors during a trial is presented, and the calculations suggest that the stressed rats are capable of learning the maze, but that their baseline behavior hinders the process. Since both stress and long-term potentiation (LTP) increase classical conditioning and both delay the acquisition of spatial information, these results are consistent with the hypothesis that stress is inducing a phenomenon similar to, if not the same as, LTP.
\end{abstract}

In searching for biological substrates of learning, neurobiologists and behaviorists alike have focused on a long-lasting increase in synaptic efficacy in response to high-frequency stimulation: long-term potentiation, or LTP (Bliss \& Lomo, 1973). The vast majority of studies have been concentrated on the mechanism responsible for the induction and long-term expression of the response, and there is a growing consensus in the field that elucidation of that mechanism is forthcoming. What remains to be revealed is the empirical proof that LTP is indeed related to learning and memory processes in the behaving animal. Although several attempts have been made to correlate LTP with behavior, the results have been seemingly discrepant. For example, in one study, the induction of LTP increased learning of the classically conditioned eyeblink response (Berger, 1984), whereas in another, a similar induction impaired an animal's ability to perform in a spatial maze task (McNaughton, Barnes, Rao, Baldwin, \& Rasmussen, 1986). The reason for this discrepancy is unknown, but these findings suggest that LTP's effects on learning may ultimately depend on the type of learning that is assessed.

Stress can have profound consequences on learning and memory processes. These effects can be either negative or positive, and, as in the case of LTP, they can vary considerably, depending on the type of learning assessed. In the case of classical conditioning, exposure to inescapable shock dramatically increases acquisition of the eyeblink response (Shors, Weiss, \& Thompson, 1992), much as LTP does (Berger, 1984). Exposure to the same stressor

This research was supported by grants from the Whitehall Foundation, the McDonnell-Pew Program in Cognitive Neuroscience, and the office of Naval Research to T.J.S. We also thank Byron Campbell for the use of his maze. Correspondence should be addressed to T. J. Shors, Department of Psychology, Green Hall, Princeton University, Princeton, NJ 08544-1010. impairs LTP (Foy, Stanton, Levine, \& Thompson, 1987; Shors, Foy, Levine, \& Thompson, 1990; Shors, Seib, Levine, \& Thompson, 1989) and increases the affinity of the $\alpha$-amino-3-hydroxy-5-methyl-4-isoxazolepropionate (AMPA)/quisqualate receptor (Shors, Tocco, Baudry, \& Thompson, 1991; Tocco, Shors, Baudry, \& Thompson, 1991), an increase notably similar to that observed in response to LTP (Tocco, Maren, Shors, Baudry, \& Thompson, 1992). Because AMPA binding is increased with both stress and LTP, and classical conditioning is increased with both stress and LTP, it is hypothesized that stress and LTP are inducing a similar phenomenon or, at the very least, that they are converging on similar neuronal mechanisms. This hypothesis would also explain how stress impairs LTP (Shors \& Thompson, 1992): LTP or something similar has already been induced. If this hypothesis is correct, stress should impair the acquisition of spatial information in the same way that LTP does (McNaughton et al., 1986). To test this hypothesis, rats were exposed to the same regime of stress that had facilitated classical conditioning (Shors et al., 1992), but instead they were tested on the acquisition of spatial information in an eight-arm radial maze.

\section{METHOD}

\section{Subjects}

Sixteen 2-month-old Sprague-Dawley rats (220-240 g) were housed in a temperature-controlled 16:8-h light:dark environment and were provided with unlimited access to water and limited access to food during the $48 \mathrm{~h}$ preceding the first trial. Half of these animals (8) composed the stressed group, while the other half served as unstressed controls (8); the stressed and the control animals were housed separately, in groups of 2 or 3.

\section{Apparatus}

The apparatus was an elevated $(72-\mathrm{cm})$ eight-arm radial maze made of flat gray-painted wood and transparent plastic walls. The 
central platform was $33 \mathrm{~cm}$ in diameter, and each arm measured $54 \times 12 \mathrm{~cm}$. The extremity of each arm was baited with a food pellet placed in a depressed circular hole. Dim illumination was provided by a stationary $20-\mathrm{W}$ halogen bulb. Prominent posters were displayed on three sides and a black and white curtain with a bold pattern constituted the fourth. In addition, a stationary manual timer was used as a spatio-auditory beacon. The rats were observed through an eyehole in the curtain.

\section{Procedure: Initial Stressor and Habituation}

Twenty-four hours before the first trial, all animals were habituated to the maze, which was unbaited and placed outside the experimental room. They were left to explore the maze for $15 \mathrm{~min}$. The control animals were returned to their home cages. The stressed animals were placed in a restraining tube, within a black soundproof chamber; a plastic ring with two metal leads was attached to their tails and the rats were exposed to $901-\mathrm{mA}$ shocks, each lasting $1 \mathrm{sec}$, delivered at fixed intervals of $1 \mathrm{~min}$ for $90 \mathrm{~min}$.

\section{Maze Training}

Immediately preceding the first trial, the stressed rats were given five $1-\mathrm{mA}, 1-\mathrm{sec}$ shocks, $1 \mathrm{~min}$ apart for $5 \mathrm{~min}$. They were kept in the soundproof chamber for $\mathbf{3 0} \mathrm{min}$ and then placed in the maze for $15 \mathrm{~min}$, or until all eight pellets were consumed, whichever came first. The control animals were placed directly in the maze. All rats were deposited onto the central platform in a random orientation. The maze was thoroughly cleansed with $10 \%$ alcohol and manually rebaited after each trial; it was also periodically rotated by $45^{\circ}$ clockwise. Each animal underwent two daily trials, during the second and fifth eighths of the illuminated $16 \mathrm{~h}$, for 6 consecutive days. Across days, the order of training was counterbalanced between rats.

\section{Statistical Analysis}

The number of food pellets consumed, arms entered, and errors committed (an entry into a previously debaited arm constituted an error) were recorded for each trial. From these data, the animal's activity (defined as the number of arms entered, divided by the time spent in the maze), degree of perseveration (defined as the preferential reentry into a previously debaited arm), and acquisition index (as described below) were calculated. An analysis of variance (ANOVA) was performed, with one repeated measures factor (days of training).

\section{RESULTS}

\section{Errors}

We have defined an error as the entry into a previously debaited arm, rather than as the entry into a previously explored arm (a more typical definition). Previous exposure to inescapable tailshock and restraint resulted in a significantly different distribution of errors across days for the stressed group, relative to the unstressed controls $[F(5,70)=3.39, p<.01]$ (Figure 1). Initially, the unstressed rats committed more errors than did the stressed animals, since the former were actively exploring the maze while the latter were comparatively immobile (Figure 2). The mean ( $\pm S E M$ ) number of errors for the unstressed group on Day 1 was $5 \pm 3$; it was $2 \pm 1$ for the stressed group. As the unstressed rats began to learn the maze (obtain the most pellets while committing the lowest number of errors in the least amount of time), they incurred a decreasing number of errors, while the stressed rats were beginning to explore the maze and were thereby committing an increasing number of errors. The un-

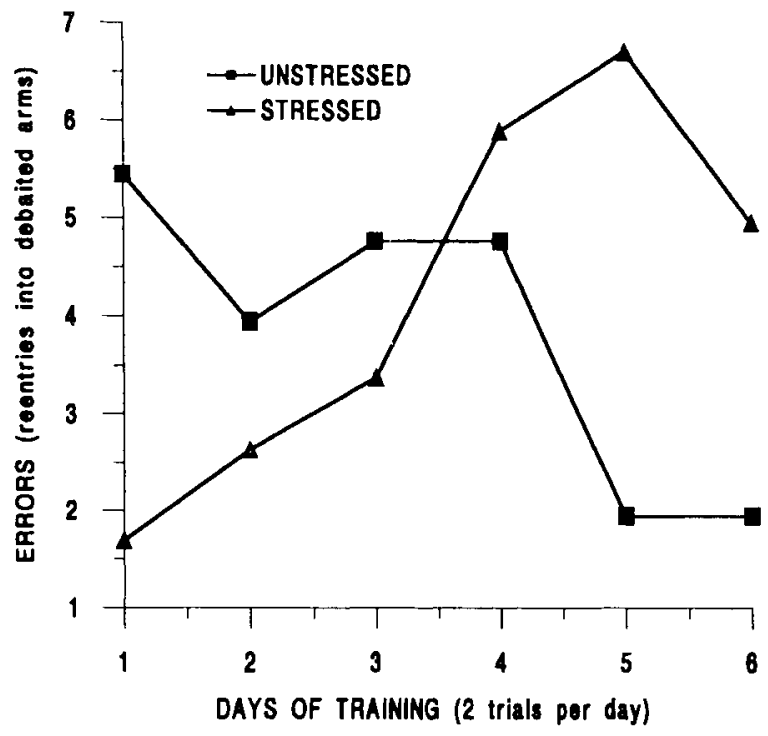

Figure 1. Mean number of errors across days for stressed $(n=8)$ and unstressed $(n=8)$ rats.

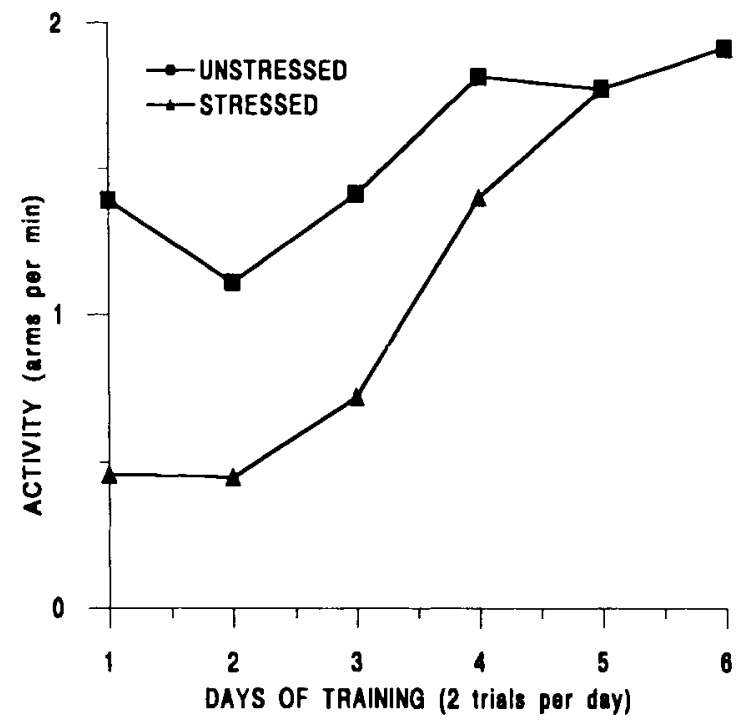

Figure 2. Mean activity levels across days for stressed and unstressed rats.

stressed rats had optimized their performance by Day 5 , and while the stressed and unstressed animals exhibited similar levels of activity at this time, the unstressed rats committed an average of only two errors per trial. On Day 6, the unstressed rats also acquired only $2 \pm 1$ errors, while the stressed group incurred $5 \pm 3$ errors.

\section{Activity}

Activity was measured by dividing the number of arms entered during a trial by the time spent in the maze (maximum $15 \mathrm{~min}$ ). Stress significantly altered activity levels: the stressed rats entered fewer arms per trial than did the 
unstressed controls $[F(5,70)=3.49, p<.01]$ (Figure 2). Although the activity deficit of the stressed animals was present only during the first 3 days and disappeared by Days 5 and 6, this early deficit in exploratory behavior could account, at least in part, for their delay in maze acquisition.

\section{Acquisition Index Equation}

In addition to the more traditional measures of maze performance, we also developed an equation to rate the degree of maze acquisition demonstrated by a rat during a single session. The equation yields a numerical evaluation of the rat's performance known as the acquisition index (A.I.):

A.I. $=\left[\sum_{i=1}^{\text {pellets }}(i-1)-\right.$ errors $\left.\cdot \frac{\sum_{i=1}^{\text {pellets }}(8-i) i}{\sum_{i=1}^{\text {pellets }} i}\right] \cdot\left(\frac{15}{\text { time }}\right)^{t}$

This equation is based on two notions. The first is that maze acquisition should be inferred from both the number of pellets consumed and the number of errors committed, and that it cannot be accurately derived from either parameter considered separately. The number of pellets consumed alone is not a foolproof indicator of acquisition, since a randomly moving rat entering 22 arms would be expected to explore each arm at least once and consume 8 pellets, even though it would not have learned the spatial task. Neither are errors, considered alone, unambiguously correlated with acquisition, since a motionless rat is neither learning nor committing errors. Both errors committed and pellets consumed must be taken into account in the evaluation of the degree of radial arm maze acquisition.

The second notion underlying the A.I. equation is the fact that the probability of making a correct or incorrect arm choice varies as a function of the number of debaited arms. For example, it is impossible for the rat to make an error when it is initially placed in the maze; however, a randomly moving rat will be seven times more likely to commit an error than to choose a correct arm when only one arm remains baited (and the seven other arms are debaited). An acquisition formula such as 8 - pellets consumed + errors, in which a more positive score reflects a lower level of spatial maze acquisition, does not take the changing probability of correct or incorrect choices into account; for example, it attributes a lower level of acquistion to a rat eating eight pellets and making nine errors (9) than to a rat eating three pellets and making one error (6).

To control for the changing probability of correct choices and errors, an increasing number of points is given for each pellet consumed: 0 points for the first pellet, 1 for the second, 2 for the third, and so on. Thus, the points earned if $i$ pellets are consumed sum to $\Sigma(i-1)$, where $i$ varies from 1 to the number of pellets consumed. Second, points are subtracted for the amount of errors com- mitted. An error occurring when only one arm has been debaited incurs -7 points, while an error occurring when seven arms have been debaited incurs -1 point.

The calculation of the negative points incurred through errors is more complex than that for the positive points received through pellet consumption, because the severity of an error depends on the number of debaited arms present when the error has occurred. A formula was devised to estimate the expected number of negative points per error, given the total number of pellets consumed during a trial. This formula is based on the logic that an error occurring when two arms have been debaited (costing -6 points) is twice as likely as one occurring when only one arm has been debaited (costing -7 points); while one costing -5 points is three times as likely, and so on.

According to the A.I. equation, a randomly moving rat will acquire an A.I. score of approximately 0 , regardless of the number of arms entered and pellets consumed, whereas a rat that is deliberately returning to a previously debaited arm will acquire a negative A.I. score. A positive score, however, indicates a nonrandom behavior directed toward previously unentered arms.

The A.I. equation described so far does not take the time the rat takes to complete the maze into account. For example, it does not attribute a higher score to a rat completing the maze in $5 \mathrm{~min}$ rather than $14.5 \mathrm{~min}$ (provided that they commit the same number of errors). The equation is, however, not completely insensitive to activity levels (defined as arms over time). Indeed, a rat that demonstrates a certain degree of maze acquisition (i.e., its choice of correct arms is above chance levels) will tend to increase its A.I. score with every choice; conversely, a rat that perseverates (deliberately returns to a previously debaited arm) will get an increasingly negative score as its activity level increases. In summary, the A.I. equation is intrinsically activity dependent until the rat actually completes the maze (i.e., until it consumes eight pellets in less than the $15 \mathrm{~min}$ ); after that, the equation is sensitive to errors, and not to the rat's velocity or speed. Time can be taken into account by multiplying the present A.I. score by $(15 / \text { time })^{t}$, where $t$ represents a time factor that can be adjusted to reflect the importance of time in maze performance. For example, if $t=0$, no relevance is attributed to the time of maze completion; whereas if $t=0.5$, a 4-min maze completion is considered twice as efficient in terms of maze acquisition as is a 15-min completion (errors being equal).

\section{Acquisition Indices: Results}

The ANOVA of the acquisition indices revealed no significant difference across days between stressed and unstressed rats, with both 0 and 0.5 used as time factors ( $p=.73$ and .36 , respectively), suggesting that exposure to the stressor did not have a significant impact on the rate of spatial learning; that is, stressed rats were capable of acquiring the radial arm maze. On any given day, however, the acquisition indices of the stressed animals were significantly lower than those of the unstressed animals. With a time factor of 0 , which attributes no rele- 
vance to the time of maze completion, the effect of stress alone was at the .05 level of probability $[F(1,14)=4.53$, $p=.05$ ], while with a time factor of 0.5 , in which a 4-min maze completion is considered twice as effective as a 15-min completion, the effect of stress alone was significant $[F(1,14)=4.93, p=.04]$ (Figure 3). In total, then, the stressed animals were capable of learning the maze and at a rate not significantly different from that of the unstressed controls. Their baseline performance, however, was significantly impaired, and this was not entirely attributable to changes in activity.

\section{Perseveration}

Initially, a rat will repeat a behavior for which it has been rewarded; it will therefore reenter an arm in which it has found a food pellet until it learns that the arms are not rebaited. In the context of this experiment, the preferential entry into previously debaited arms is represented by a negative A.I. score, which was considered a sign of perseveration. As is shown in Figure 3, both the stressed and the unstressed rats demonstrated an initial degree of perseveration on Days 1 and 2, but the stressed and unstressed rats differed in that the former manifested a greater degree of perseveration and the behavior lasted for a greater length of time.

\section{DISCUSSION}

Since both stress and LTP increase AMPA binding (Shors et al., 1991; Tocco et al., 1991, 1992) and classical conditioning (Berger, 1984; Shors et al., in press), and since the induction of either impairs the subsequent expression of LTP (Bliss \& Lomo, 1973; Shors \& Thompson, 1992), we hypothesized that stress and LTP act through similar neuronal mechanisms. If such a hypoth-

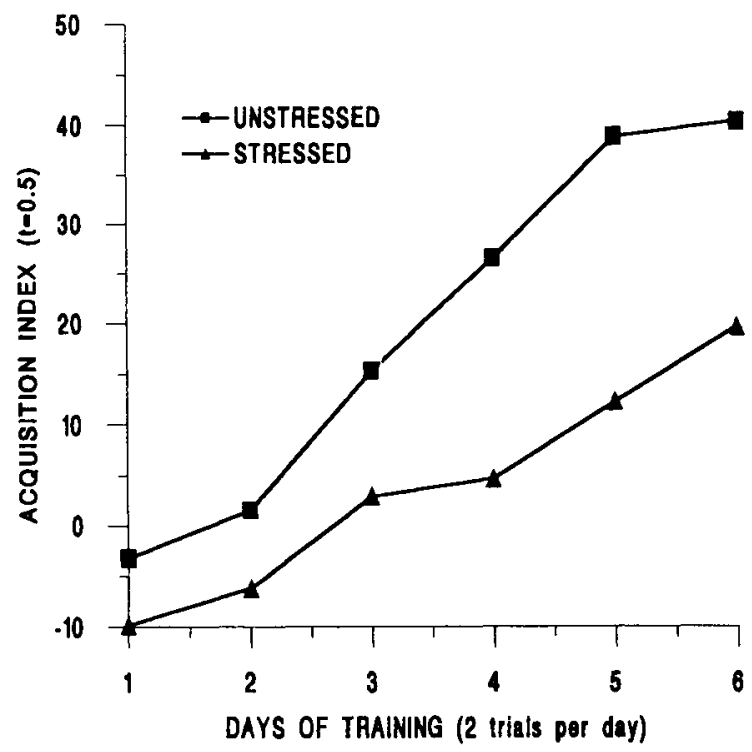

Figure 3. Mean acquisition indices for stressed and unstressed rats. Values were derived from the acquisition index equation, using a time factor of 0.05 . esis were valid, stress should impair the acquisition of spatial information, much in the same way as LTP does (McNaughton et al., 1986). Indeed, as reported here, exposure to a stressor patterned after that used to induce learned helplessness (Overmier \& Seligman, 1967) and helpfulness (Shors et al., 1992) resulted in a delay in the rat's ability to acquire the most efficient radial arm maze strategy. This effect was demonstrated by a significantly different distribution of errors across days between the stressed and the unstressed rats (Figure 1). The two factors contributing to the delay in acquisition include the stressed rats' initial decrease in exploratory behavior and their greater tendency to perseverate by entering the arms they first entered.

Overall, the most likely contributor to the delayed acquisition observed in the stressed rats was their initial low level of exploratory behavior. In terms of activity (number of arms entered over time), the stressed animals initially exhibited an activity deficit in comparison with the unstressed animals, although their activity levels increased by Day 3, reaching unstressed levels by Days 5 and 6 (Figure 2). Similarly, during the first 3 days (six trials) of training, the stressed rats committed fewer errors than did the unstressed controls, since the stressed rats were not as active in exploring the maze. By the 10th session, however, the stressed rats were committing more errors than were the unstressed controls, since by then the latter had learned the maze and were navigating through it more efficiently than the stressed rats.

In addition to the more standard measures of maze performance, we also present an equation that evaluates the performance of a rat during a single session and yields an A.I. (i.e., a numerical score). This equation takes into account the number of pellets consumed, the number of errors committed, the changing probability of making a correct or an incorrect arm choice, and, to a varying extent, the degree of activity. The results obtained through this formula suggest that the stressed rats were able to acquire the correct radial arm maze strategy, at a rate comparable to that of the unstressed animals. Therefore, the overall rate of spatial acquisition was not impaired by stress under the present experimental conditions. The mean acquisition indices of the stressed rats, however, were lower than those of the unstressed animals on any given day, and the initial acquisition levels of the stressed and unstressed animals indicate that the former were perseverating more and for a longer period of time than the latter. Since the acquisition index with a time factor of 0 allocates no inherent benefit to speed or velocity, and since the difference between the stressed and the unstressed rats was at the $p=.05$ level of significance, it seems that stress has an impact on the acquisition of spatial information that is not entirely attributable to changes in activity.

Given that a rat will repeat a behavior that leads to a reward, it is understandable that both the stressed and the unstressed animals manifest an initial degree of perseveration (see Figure 3, Days 1 and 2; a negative A.I. score reflects perseveration). Through their exploration of the 
maze, however, the animals soon learn that perseveration is unrewarded, and one would expect that animals with high activity levels would learn more quickly not to perseverate than would rats with low activity. Consequently, it is possible that the higher degree of perseveration demonstrated by the stressed animals relative to the unstressed rats is partly due to the former's lower activity levels. It is also reasonable to assume that a stressed rat, having just experienced a relatively traumatic event, would return to an arm that was previously deemed safe. A more direct, neurological cause for the stressed group's higher degree of perseveration, however, cannot be ruled out. In any case, such a tendency to return to a specific arm suggests that the rats are using spatial cues despite exposure to the stressor, and therefore stress is not acting as a functional hippocampal lesion. In general, hippocampal lesions tend to increase activity and perseveration-type (win-stay) behavior (Gray \& McNaughton, 1983; Packard, Hirsh, \& White, 1989), yet decrease exploratory behavior (Thinus-Blanc, Save, Poucet, \& Buhot, 1991).

In the controversy surrounding learned helplessness, two general theories have emerged. One theory is cognitive in nature and asserts that during exposure to inescapable shock, the rat learns that the shock and the rat's response pattern are not contingent; the rat is thereafter unable to form the relevant contingencies (Maier \& Seligman, 1976; Overmier \& Seligman, 1967). The other theory attributes the performance decrement to the animal's ensuing inactivity, which results either from the association of the shock with the rat's inactive response pattern and/or neurotransmitter depletion (Anisman, de Catanzaro, \& Remington, 1978; Glazer \& Weiss, 1976). Several studies have dissociated inactivity from associational deficits (Jackson, Alexander, \& Maier, 1980; Maier \& Jackson, 1979) and thereby substantiate the theory that inactivity cannot fully account for the learning deficits, as our results from using the A.I. index indicate. Nonetheless, when movement is critical to learning (as in the present study), the relative contribution of decreased activity levels to subsequent learning deficits is difficult if not impossible to assess.

Unfortunately, many of the behavioral studies in which LTP has been used as an independent variable fail to mention motor effects, and therefore, we cannot make a direct comparison between the effects of stress and LTP on activity and their respective contribution to the stress-induced impairment in the acquisition of spatial information. Although such an omission may simply signify that there is no effect, there is ample evidence to suggest that LTP can modulate movement. For example, the most effective stimulus for the induction of LTP consists of a tetanus delivered at theta (Larson, Wong, \& Lynch, 1986), which corresponds to an endogenous rhythm associated with voluntary movement (Fox, Wolfson, \& Ranck, 1986; Vanderwolf, 1969) and exploration of new environments (Hill, 1978). Moreover, the endogenous rhythm is compromised in animals exposed to inescapable shock (Balleine \& Cur- thoys, 1991). In addition, LTP-like phenomena have been reported as occurring during the exploration of novel environments, but they are absent in the presence of NMDA receptor antagonists, which block LTP (Croll, Sharp, \& Bostock, 1992; Sharp, McNaughton, \& Barnes, 1989).

The difficulty of parceling out changes in activity from those specific to learning is not unique to the stress literature and is reminiscent of that discussed regarding the effect of NMDA receptor antagonists on learning (Hargreaves \& Cain, 1992; Keith \& Rudy, 1990). These drugs not only block LTP (Collingridge, Kehl, \& McLennan, 1983) and spatial maze learning (Morris, Anderson, Lynch, \& Baudry, 1986), but also are notorious for their significant impact on movement and motivation. Aside from classical conditioning, few learning paradigms allow for the independent measurement of the exact movement without the learning superimposed. Furthermore, few tasks exist in the laboratory setting that do not require some type of deprivation or punishment in order to motivate the animal to learn. If we consider that motivation and stress, of some sort, are inherent in the learning situation, and that these two factors, in and of themselves, can affect exploratory behavior, the fact that stress effects on learning can, at least in part, be attributed to alterations in exploration does not negate the fact that stress can have a major impact on learning. To the contrary, in many cases, learning would not occur without it.

Regardless of the reasons for the impairment in the acquisition of spatial information, our primary aim was to determine whether the impairment would occur in response to stress, as it did in response to the induction of LTP. McNaughton et al. (1986) performed four experiments to investigate the retrograde and anterograde effects of LTP induction on spatial memory. They used two spatial tasks: the circular platform task, which obliges the animal to use spatial cues to find a darkened escape tunnel, and the eight-arm radial maze. They found that the induction of LTP does not affect information previously acquired in either spatial task, but that LTP-induced animals have more difficulty adapting to a new tunnel location in the reacquisition of the circular platform task than do control animals; this impairment was reflected by increased levels of errors in the LTP-induced group and the degrees of deviation from the tunnel location. Both of these parameters, however, indicated that the LTPinduced animals demonstrated a substantial improvement across days and therefore were able to learn the task; the LTP-induced animals differed from the controls mainly in their initial inability to respond to the novel location of the escape tunnel and their persistence in returning to the previous tunnel location, symptoms of perseveration.

Corresponding results were found in the present study: while stress impaired the acquisition of the radial arm maze and baseline performance, the stressed animals were capable of learning the task and demonstrated unimpaired learning rates. These observations are also consistent with previous studies in which exposure to inescapable shock 
resulted in an increase in perseveration toward an illuminated arm in a water maze (Szostak \& Anisman, 1985), an increase that did not significantly impair choice accuracy. We are inclined to agree with these authors' view that stress invokes in the animal a tendency to return to innate defensive responses; but that once the animal learns that the particular response is not rewarded, the animal can and will learn the relevant contingency.

Neither the induction of LTP (Corol, Abel, Church, Barnes, \& McNaughton, 1992) nor exposure to restraint impair performance in the Morris water maze (Warren, Castro, Rudy, \& Maier, 1991). Although the eight-arm radial maze task is similar to the Morris water maze in its hippocampal dependence (Morris, Garrud, Rawlins, \& O'Keefe, 1982; Morris et al., 1986), it is obviously much less stressful. To motivate the rats to find the food pellets, they are partially food deprived and are therefore only moderately stressed relative to rats forced to swim. Perhaps tasks that are predicated on high stress are not as susceptible to additional stress, although the authors rightly point out that robust helplessness effects have been obtained in other stressful tasks, such as escape and avoidance training (Maier \& Jackson, 1979; Maier \& Seligman, 1976). Alternatively, if the activity deficit is contributing to our delay in acquisition, the forced swimming procedure used in the Morris water maze may overcome the activity deficit, leaving acquisition unaffected. In fact, in the presence of ordinarily aversive cues, inescapable shock can actually increase vigorous swimming activity (Szostak \& Anisman, 1985).

The identical regime of stress used in the present study resulted in a robust facilitation of classical eyeblink conditioning in the freely moving rat (Shors et al., 1992), a phenomenon that was termed learned helpfulness. This increase in learning stands in contrast to standard helplessness effects and the effect reported here, and it paradoxically supports our hypothesis that stress is inducing a form of synaptic potentiation similar to LTP. As mentioned, LTP also increases learning of this task (Berger, 1984), and learning is accompanied by an increase in the monosynaptic evoked response in the dentate gyrus, an increase resembling LTP (Weisz, Clark, \& Thompson, 1984).

Given that the stressor and/or LTP induction are kept constant, the variables responsible for facilitation in one case and impairment in another must be the type of learning that is being assessed and/or the neural structures subserving that learning. Although much of learning may ultimately depend on distributed systems throughout the brain, the reported stress- and LTP-induced effects on learning were demonstrated in tasks that have been localized to specific areas of the brain. In the case of classical conditioning, there is overwhelming evidence that the essential circuitry for the delay eyeblink paradigm is located in the brainstem and cerebellum (McCormick \& Thompson, 1984; Thompson, 1990), whereas in the case of spatial maze learning, the hippocampus is essential (Jarrad, 1978; Olton, Walker, \& Gage, 1978; Olton, Walker, \& Wolf, 1982; O'Keefe \& Nadel, 1978). Since both the stress- and LTP-induced increases in learning were demonstrated in a task that is not dependent on the hippocampus, it is conceivable that learning is impaired when the task is dependent on the hippocampus, as reported here, but not impaired (or even facilitated) when it is dependent on other structures. Such an explanation has its complications, since helplessness effects are not limited to hippocampal-dependent tasks. It is worth noting that most helplessness effects have been demonstrated in instrumental as opposed to classical conditioning.

\section{CONCLUSION}

Our overall hypothesis is that LTP is a mechanism that modulates neural resources devoted to learning complex contingencies under conditions of stress. The specific hypothesis that stress induces LTP or something very similar would explain how stress impairs LTP (LTP has already been induced), and it is supported by the fact that both stress and LTP increase AMPA binding, both increase classical conditioning, and both impair the acquisition of spatial information. The behavioral linkage suggests that LTP does have consequences for the behaving organism. The difference in the direction of the effects of LTP and stress on learning may be attributable to specific task demands, demands that engage the hippocampus in varying, but presumably predictable, degrees. In conclusion, since stress impairs the acquisition of spatial information in much the same way as the induction of LTP does, one more piece of evidence stands to support similar roles for LTP and stress in learning.

\section{REFERENCES}

Anisman, H., de Catanzaro, D., Remington, G. (1978). Escape performance following exposure to inescapable shock: Deficits in motor response maintenance. Joumal of Experimental Psychology: Animal Behavior Processes, 4, 197-218.

Balleine, B. W., \& Curthoys, I. S. (1991). Differential effects of escapable and inescapable footshock on hippocampal theta activity Behavioral Neuroscience, 105, 202-209.

BERGER, T. W. (1984). Long-term potentiation of hippocampal synaptic transmission affects rate of behavioral learning. Science, 224 $627-630$.

Buss, T. V. P., \& LoMo, T. (1973). Long-lasting potentiation of synaptic transmission in the dentate gyrus of the anesthetized rabbit following stimulation of the perforant path. Journal of Physiology, 232, 331-356.

Collingridge, G. L., Kehl, S. J., \& Mclennan, H. (1983). Excitatory amino acids in synaptic transmission in the Schaeffercommissural pathway of the rat hippocampus. Joumal of Physiology, $443,33-46$

Corol, D. L., Abel, T. W., Church, L. T., Barnes, C. A., \& MCNAughton, B. L. (1992). The saturation of long-term enhancement of perforant path synapses impair spatial learning in the Morris water task? A failure to replicate. Society for Neuroscience Abstracts, $18,1217$.

Croll, S. D., Sharp, P. E., \& Bostock, E. (1992). Evidence for NMDA receptor involvement in environmentally induced dentate gyrus plasticity. Hippocampus, 2, 23-28.

Fox, S. E., Wolfson, S., \& RANCK, J. B. (1986). Hippocampal theta rhythm and the firing of neurons in walking and urethane anesthetized rats. Experimental Brain Research, 62, 495-508. 
Foy, M. R., Stanton, M. E., Levine, S., \& Thompson, R. F. (1987) Behavioral stress impairs long-term potentiation in rodent hippocampus. Behavioral \& Neural Biology, 48, 138-149.

Glazer, H. I., \& Weiss, J. M. (1976). Long-term and transitory interference effects. Joumal of Experimental Psychology: Animal Behavior Processes, 2, 191-201.

Gray, J. A., McNaughton, N. (1983). Comparison between the behavioral effects of septal and hippocampal lesions: A review. Neuroscience \& Biobehavioral Reviews, 7, 119-188.

Hargreaves, E. L., \& Cain, D. P. (1992). Hyperactivity, hyporeactivity, and sensorimotor deficits induced by low doses of the $\mathrm{N}$-methyl-D-aspartate non-competitive channel blocker MK801. Behavioral Brain Research, 47, 23-33.

HILL, A. J. (1978). First occurrence of hippocampal spatial firing in a new environment. Experimental Neurology, 62, 282-297.

Jackson, R. L., Alexander, J. H., Maier, S. F. (1980). Learned helplessness, inactivity, and associative deficits: Effects of inescapable shock on response choice escape learning. Joumal of Experimental Psychology: Animal Behavior Processes, 6, 1-20.

JARRAD, L. E. (1978). Selective hippocampal lesions: Differential effects on performance by rats of a spatial task with preoperative versus postoperative training. Journal of Comparative \& Physiological Psychology, 92, 1119-1127.

KeITH, J. R., RuDY, J. W. (1990). Why NMDA-receptor-dependent long-term potentiation may not be a mechanism of learning and memory: Reappraisal of the NMDA-receptor blockade strategy. Psychobiology, 18, 251-257.

Larson, J., WONG, D., \& LYNCH, G. (1986). Patterned stimulation at the theta frequency is optimal for the induction of hippocampal longterm potentiation. Brain Research, 368, 347-350.

Maier, S. F., JACKSON, R. L. (1979). Learned helplessness: All of us were right (and wrong). Inescapable shock has multiple effects. Psychology of Learning \& Motivation, 13, 155-218.

Maier, S. F., Seligman, M. E. P. (1976). Learned helplessness: Theory and evidence. Journal of Experimental Psychology: General, 105, 3-46.

McCormick, D. A., Thompson, R.F. (1984). Cerebellum: Essential involvement in the classically conditioned eyeblink response. Science, 223, 296-299.

McNaughton, B. L., BARnes, C. A., RAo, G., Baldwin, J., \& RasMUSSEN, M. (1986). Long-term enhancement of hippocampal synaptic transmission and the acquisition of spatial information. Joumal of Neuroscience, 6, 563-571.

Morris, R. G. M., Anderson, E., Lynch, G. S., \& Baudry, M. (1986). Selective impairment of learning and blockade of long-term potentiation by $\mathrm{N}$-methyl-D-aspartate receptor antagonist, AP-5. Nature, 319, 744-776.

Morris, R. G. M., Garrudd, P., RAwlins, J. N. P., \& O'Keefe, J. (1982). Place navigation impaired in rats with hippocampal lesions. Nature, 297, 681-683.

O'KeEFE, J., * NADEL, L. (1978). The hippocampus as a cognitive map. London: Oxford University Press.

Olton, D. S., Walker, J. A., \& GAge, F. H. (1978). Hippocampal connections and spatial discrimination. Brain Research, 139, 295-308.

Olton, D. S., Walker, J. A., Wolf, W. A. (1982). A disconnection analysis of hippocampal function. Brain Research, 233, 241-253.
Overmier, J. B., Seligman, M. E. P. (1967). Effects of inescapable shock upon subsequent escape and avoidance learning. Journal of Comparative \& Physiological Psychology, 63, 23-33.

PaCkard, M. G., Hirsh, R., \& Wite, N. M. (1989). Differential effects of fornix and caudate lesions on two radial maze tasks: Evidence for multiple memory systems. Joumal of Neuroscience, 9, 1465-1472.

Sharp, P. E., McNaughton, B. L., * Barnes, C. A. (1989). Exploration-dependent modulation of evoked responses in fascia dentata: Fundamental observations and time course. Psychobiology, 17 257-269.

Shors, T. J., Foy, M. R., Levine, S., a Thompson, R. F. (1990). Unpredictable and uncontrollable stress impairs neuronal plasticity in the rat hippocampus. Brain Research Bullesin, 24, 663-667.

Shors, T. J., Seib, T. B., Levine, S., Thompson, R. F. (1989). Inescapable versus escapable shock modulates long-term potentiation (LTP) in rat hippocampus. Science, 244, 224-226.

Shors, T. J., \& Thompson, R. F. (1992). Stress impairs (or induces) synaptic LTP, but does not affect paired-pulsed facilitation in the stratum radiatum of the rat hippocampus. Synapse, 11, 262-265.

Shors, T. J., Tocco, G., Baudry, M., a Thompson, R. F. (1991). Acute stress increases AMPA binding to the AMPA/quisqualate receptor and the increase is not glucocorticoid-dependent. Society for Neuroscience Abstracts, 17, 915 .

Shors, T. J., Weiss, C., \& Thompson, R. F. (1992). Stress-induced facilitation of classical conditioning. Science, 257, 537-539.

Szostak, C., ANISMAN, H. (1985). Stimulus perseveration in a water maze following exposure to controllable and uncontrollable shock. Behavioral \& Neural Biology, 43, 178-198.

Thinus-Blanc, C., Save, E., Poucet, B., Buhot, M. C. (1991). The effects of reversible inactivations of the hippocampus on exploratory activity and spatial memory. Hippocampus, 1, 365-372.

Tномpson, R. F. (1990). Neural mechanisms of classical conditioning in mammals. Philosophical Transactions of the Royal Society of London, 329, 161-170.

Tocco, G., Maren, S., Shors, T. J., Baudry, M., * Thompson, R. F. (1992). Long-term potentiation is associated with increased [H]AMPA binding in rat hippocampus. Brain Research, 573, 228-234.

Tocco, G., Shors, T. J., BAudry, M., Thompson, R. F. (1991). Selective increase of AMPA binding to the AMPA/quisqualate receptor in the hippocampus in response to acute stress. Brain Research, 559, 168-171.

VANDERWOLF, C. H. (1969). Hippocampal electrical activity and voluntary movement in the rat. Electroencephalography \& Clinical Neurophysiology, 26, 407-418.

Warren, D. A., Castro, C. A., Rudy, J. W., Majer, S. F. (1991) No spatial learning impairment following exposure to inescapable shock. Psychobiology, 19, 127-134.

Weisz, D. J., Clark, G. A., Thompson, R. F. (1984). Increased responsivity of dentate granule cells during nictitating membrane response conditioning in the rabbit. Behavioral Brain Research, 12 , 145-154.

(Manuscript received June 26, 1992; revision accepted for publication July 16, 1992.) 